\title{
Les discours du tourisme en ligne et hors-ligne : questions d'analyse et réflexions théoriques
}

\section{Online and offline tourism discourses: analytical questions and theoretical reflections}

\author{
Glória Abreu França ${ }^{1, a}$ \\ ${ }^{1}$ Université Paris 13 Sorbonne Paris Cité, 99, Avenue Jean Baptiste Clément - Villetaneuse - France
}

\begin{abstract}
Résumé. Ce texte propose une réflexion sur le parcours méthodologique que j'ai effectué à partir d'un changement de perspective d'analyse dans mon travail sur les discours du tourisme sur le Brésil. La notion de «genre de discours» (les guides touristiques peuvent en être un exemple) a été choisie au départ comme un des critères pour la sélection du corpus, et a en effet servi comme un outil heuristique au début du travail de construction du corpus. Néanmoins, dans un deuxième moment, le travail analytique a débouché sur la notion de «trajet thématique » qui a dès lors guidé la constitution du corpus. Le thème des «voyages au Brésil lors de la Coupe du Monde » est devenu l'organisateur du corpus qui a alors été constitué dans une double hétérogénéité. À partir de cette configuration thématique, j'ai pu explorer dans les analyses des trajets des discours et de leurs sens autour de questions telles que le tourisme sexuel et la prostitution, liées au thème de la Coupe du Monde au Brésil.
\end{abstract}

\begin{abstract}
This text reflects on the methodological approach, in my research about tourism discourses about Brazil, set up from an analytical input change. The "discourse genre » concept (touristic guides can be an example of it) was established at the beginning as one of the criteria for the corpus selection, and has, as a matter of fact, worked as a heuristic tool by the beginning of the corpus construction. In a second moment, however, the analytical work led to the «thematic route » notion, which has guided the corpus constitution since then. The «Trips to Brazil during the World Cup» theme has organized the corpus, which is configured in a double heterogeneity. From this thematic configuration, I could follow in the analysis the meanings paths about issues, as sexual tourism and prostitution, linked to the World Cup in Brazil issue.
\end{abstract}

\section{Introduction}

Ce texte porte sur le parcours théorico-méthodologique effectué au cours de mon travail de thèse, dans le cadre de l'analyse du discours de courant matérialiste. L'élaboration finale du cadre méthodologique de ce travail m'a permis de comprendre les différents mouvements effectués dans la tâche d'analyse, de ressaisir le parcours réalisé et surtout de décider quelles seraient les stratégies que j'adopterai vis-à-vis de la clôture du corpus. Cet exercice se construit donc dans une perspective de réflexivité, qui va ainsi dans le même sens que l'argumentaire de ce colloque, qui proposait de réfléchir sur nos parcours en même temps que nous les construisons, mais aussi de partager des réflexions autour des enjeux méthodologiques soulevés par nos recherches. Plus spécifiquement, j'aborde ici un réaménagement théorique que j'ai effectué pendant le processus de construction de mon corpus de travail. Dans ce cadre, je mets l'accent sur le rôle d'une double hétérogénéité dans la constitution du corpus, en suivant ainsi Zoppi-Fontana (2003: 2 [1]). Je considère ainsi que le corpus

\footnotetext{
${ }^{a}$ Auteur de correspondance : gloria.franca@gmail.com
}

This is an Open Access article distributed under the terms of the Creative Commons Attribution License 4.0, which permits unrestricted use, distribution, and reproduction in any medium, provided the original work is properly cited 
constitué pour mon travail de recherche est hétérogène à deux niveaux. Le premier niveau est celui de la «matérialité symbolique», qui, dans le cas de mon travail, se montre doublement: dans les différents supports (papier/numérique) et dans la multisémioticité des discours analysés (iconiques, verbaux, techno-langagiers, etc.). Le deuxième niveau est celui de l'« inscription institutionnelle et la circulation sociale» : cette caractéristique dans mon travail prend forme (dans mon travail) dans la diversité de genres auxquels appartiennent les différentes matérialités langagières/discursives présentes dans le corpus : le site officiel du ministère du tourisme avec ses documents numériques, numériqués et numérisés ${ }^{\mathrm{b}}$ par exemple; ou encore les pages web des réseaux sociaux et les blogs de voyage liés à cet organisme, qui comportent des entretiens, des résolutions, des campagnes socioéducatives, des instructions et suggestions de voyages - plusieurs formes discursives ayant un air de famille avec les discours des guides touristiques par exemple.

Tout au long de cet article j'utilise le terme corpus pour désigner différents objets : cette plasticité dans la désignation est provisoire et nécessaire : elle suit les différents moments de la recherche, depuis la construction/constitution du corpus jusqu'à son analyse. Dans les études du discours, le corpus se définit par rapport à la notion d'archive. Le corpus est vu comme "le résultat d'un processus de construction contemporain au processus analytique, qui considère le fonctionnement de la mémoire discursive comme un principe de lisibilité des énoncés ${ }^{c} »$, tandis que l'archive est prise dans le sens large de «champs des documents pertinents et disponibles sur une question ${ }^{\mathrm{d}} »$. C'est à partir de la prise en compte de l'archive que j'ai pu identifier un thème. Je pourrais, dans ce sens, affirmer que j'ai eu affaire à une grande diversité d'archives et qu'à partir du moment où la collecte s'est mise en place, j'ai construit ma propre archive, qui, comme on le verra par la suite, a pu m'aider à éclaircir différents moments de l'analyse. À partir de cette distinction, je peux identifier trois moments dans la constitution du corpus et de l'archive. Dans un premier moment, corpus désigne plutôt une grande masse d'énoncés iconico-verbaux, qui n'est pas encore délimitée par des régularités : cette notion provisoire a plutôt le sens des «matériaux » collectés et regroupés. Dans un deuxième moment, le corpus peut être compris comme un ensemble construit par mon geste analytique et rassemblé autour d'une problématique définie: il s'agit plutôt d'une étape plutôt descriptive. En dernier lieu, à partir des gestes analytiques et interprétatifs, la conception de corpus devient plus spécifique. Dans ce dernier cas, lorsque l'étape d'analyse prend place, le corpus se rapproche de l'ensemble qui sera finalement l'objet d'analyse et d'interprétation : Moirand (2004 [9]) appelle cet ensemble «sous-corpus d'analyse », je me rapproche, pour ma part, de ce qu'Orlandi (1984 [10]) appelle « un découpage ».

\section{La notion de genre de discours: double lignée dans mon travail}

Je me suis posée la question de savoir par où je pourrais commencer mon travail en ayant comme problématique la construction et la circulation d'une doxa sur le Brésil et sur les brésilien.ne.s. J'ai donc eu recours à la notion de genre de discours, en vue de construire un corpus qui soit cohérent et qui garde une certaine routine de genre. Le genre est dans ce sens conçu plutôt comme cadres discursifs renvoyant à des formats de discours qui sont connus et reconnus, ou plus précisément « des processus discursifs dynamiques qui se sédimentent historiquement par leur circulation dans la société à cause de conditions de production déterminées » (ma traduction, Zoppi-Fontana, 2012 : 77 [11]).

\footnotetext{
${ }^{\mathrm{b}}$ cf. distinction faite par M-A Paveau, 2013 [2].

${ }^{c}$ Pêcheux, 1984 [3], apud Zoppi-Fontana, 1998 [4].

d Pêcheux, 1982 : 37 [5], apud Zoppi-Fontana, 1999 [6]. Le rôle de la notion d'archive quoique clairement centrale pour la réflexion que je développe ici ne pourra pas être exploitée directement dans ce texte, pour des questions d'espace. Je me contenterai donc de renvoyer aux extraits et aux lectures utilisées dans cet article concernant la notion, dont une des définitions est la suivante : l'archive «est un dispositif non fortuit qui constitue des figures distinctes, en ce sens que chaque dispositif d'archive établit sa propre mise en ordre. Ainsi, du côté de l'archive, le sens est convoqué à partir d'une diversité maximale de textes, de dispositifs d'archive spécifiques d'un thème, d'un événement, d'un itinéraire » (Guilhaumou, Maldidier \& Robin, 1994 : 195 [7]). (Cf. également Zoppi-Fontana, 2002 [8]).
} 
Zoppi-Fontana considère dans ce sens que les genres de discours doivent être décrits dans leurs fonctionnements et non pas dans leurs formes. A ce sujet, l'auteure formule ce qui serait une approche des genres dans une étude discursive :

« toute typologie des genres de discours qui se réduise uniquement à identifier des formes, types de textes ou situations d'utilisation est vouée à l'échec, puisque les pratiques sociales dans l'Histoire sont des processus dynamiques, changeants, sans début ni fin, ancrés dans la reprise des éléments déjà présents, mais toujours en transformation. La description et l'analyse des genres doivent faire face à cette dimension d'événement » (ma traduction, Zoppi-Fontana, $2012: 80[11])$.

Cette notion, prise dans le cadre d'une théorie du discours, dont la conception du travail méthodologique est dynamique, et surtout qui s'intéresse à des processus discursifs qui dépassent le cadre de catégories formelles préétablies avant l'analyse m'a cependant servi dans un premier moment, de catégorie de sélection et de classement des énoncés, en prenant comme point de départ l'appartenance institutionnelle (c'est le cas pour les guides de voyages écrits). Je montrerai, tout d'abord, la façon dont mes premiers points de repères ont configuré mes objectifs de recherche et mes critères méthodologiques - notamment à travers la reconnaissance d'un premier genre de discours, celui des guides du tourisme $e^{\mathrm{e}}$; deuxièmement, je parlerai du rôle de l'hétérogénéité du corpus, pour ensuite, expliciter dans cette perspective la façon dont les genres du discours ont fonctionné dans mon travail.

\subsection{Les objectifs et les critères établis}

En analyse du discours, le corpus est toujours (re)mis en question et on parle donc de construction ou constitution dynamique du corpus, sur lequel on mène une analyse en spirale, c'est-à-dire que le moment de l'analyse/interprétation influence toujours en retour la constitution du corpus. En vue d'expliciter ce parcours dynamique, je rappellerai brièvement en quoi consistait mon projet initial, en mettant l'accent sur le lien qui existe entre ma problématique et mes critères préétablis pour la construction du corpus.

Dans mon projet initial de thèse, $\mathrm{j}$ 'ai formulé plusieurs questions à partir de mon hypothèse principale de recherche, liée à la production/circulation de discours sur le Brésil et sur les Brésilien.ne.s, mais aussi concernant les lieux de circulation de ces discours. La problématique se fondait sur une comparaison de deux ordres: d'une part une comparaison au niveau de la langue/culture entre les discours qui circulent en France et au Brésil; d'autre part, une comparaison des différents environnements matériels, car le corpus incluait (en majorité) des discours provenant des différents supports : les guides de tourisme et des forums de discussions sur internet. Je traiterai ici en particulier de ce dernier point et je m'intéresserai à l'imbrication entre la constitution matérielle de mon corpus et la formulation de ma problématique.

Comme ma problématique porte sur la circulation des discours fondés sur une certaine doxa, un de mes objectifs de recherche consiste à m'interroger sur la manière dont la composition hétérogène et multi-sémiotique du corpus peut indiquer des résultats différents à propos de la circulation de ces discours doxiques. Plus précisément, je me suis posée la question de savoir s'il y a des divergences discursives et sur quoi elles pouvaient porter en ce qui concerne la formulation et la circulation des discours dans l'environnement papier (les guides de tourisme) et dans les environnements numériques (tels que les forums), discours que j'avais intégrés à mon corpus dans un premier moment. Deux de mes cinq critères méthodologiques établis dans mon projet initial étaient directement reliés à cette question : outre le critère linguistique (français/portugais), le critère chronologique (2012-2014) et le critère théorique (la gender theory), j'en ai établi deux autres: il s'agit du critère «support » (papier/numérique) et du critère «genre de discours» (dans le discours du tourisme). Ces deux critères, qui ne se confondent pas, m'ont ainsi servi, dans un premier moment, à sélectionner ce qui pourrait faire partie de la constitution du corpus.

\footnotetext{
${ }^{\mathrm{e}} \mathrm{Au}$ sujet de quelques catégorisations de ce qui serait un genre de discours du tourisme, voir Moirand (2004b [12]), «Le même et l'autre dans les guides de voyage au XXIe siècle », où l'auteure insère ce genre dans la catégorie des écrits de transmission de savoir.
} 
Comme je l'ai dit plus haut, l'avancement de mes analyses m'a amenée à élargir aussi bien les termes de ma problématique que la constitution matérielle de mon corpus, et cela va sans doute de pair avec l'approfondissement théorique auquel j'ai abouti au fil des lectures et des analyses. Sur l'aspect théorique, j'ai formulé plus explicitement la contribution des études de genre (gender theory) pour mon cadre théorique - l'analyse du discours matérialiste -, et $\mathrm{j}$ 'ai ainsi pu observer la formulation et circulation de propos portant sur le Brésil et sur les Brésilien.ne.s de manière distincte selon le critère linguistique (France/Brésil). En ce qui concerne mes premières observations sur les discours produits au Brésil, j'ai constaté que les discours dont l'analyse m'intéressait circulaient non seulement dans les guides ou dans les forums de discussions (comme j'avais pu le voir dans le cas du français), mais aussi sur les blogs de voyages, sur le site officiel du ministère du tourisme et sur les différents réseaux sociaux de cet organisme (avec ses documents de planification du tourisme dans le pays lors de la préparation des événements sportifs qui y auraient lieu - la Coupe des confédérations, en 2013, la Coupe du monde, en 2014, et les Jeux olympiques, à Rio, en 2016).

Ce changement a entrainé une modification de l'un des critères concernant la constitution matérielle du corpus : celui des genres du discours. Ce dernier a vu son rôle modifié au nom d'une autre catégorie, le trajet thématique (voir point 3), en vue de pouvoir intégrer certains propos qui circulaient dans d'autres lieux d'énonciation (Zoppi-Fontana, 2002 [13]) et dans d'autres environnements que je n'avais pas prévus au départ. Cette hétérogénéité du corpus et son appartenance à plusieurs genres, qui existaient déjà au départ et qui se sont accrues, ont pu de cette façon enrichir les possibilités d'analyse et apporter des réponses aux questions de la thèse.

\subsection{La double hétérogénéité du corpus}

En vue de réfléchir au fonctionnement de cette hétérogénéité du corpus avec lequel je travaille, je m'inspire largement des réflexions développées par Zoppi-Fontana (2003 [1]) qui s'appuie pour sa part sur des notions mises en places dans les travaux de Pêcheux (1982 [5] ; 1983a [14]), Guilhaumou, Maldidier \& Robin (1994 [7]) et Guilhaumou (2002 [16]), entre autres.

Le fait de reprendre ces lectures signifie réfléchir sur ce que l'analyse du discours dit de la constitution hétérogène du corpus; cela réaffirme la nature de l'analyse du discours en tant que discipline d'interprétation et implique de penser aussi bien le rôle du chercheur dans l'analyse que les effets de sa lecture dans l'étude ${ }^{\mathrm{f}}$. Il s'agit également de souligner la conception dynamique du corpus, qui, d'après Zoppi-Fontana $\left(2003^{\mathrm{g}}: 3-4\right.$ [1]), est une conception

« solidaire d'une théorie du discours qui s'autodéfinit en tant que discipline interprétative, cela veut dire qu'elle assume le lieu constitutif de l'interprétation dans les procédures de la description [...et prend en compte] aussi bien les déterminations historiques sur les processus discursifs que les effets du geste analytique du chercheur dans la sélection, collecte, organisation et exploitation des matériels étudiés».

Cette procédure se base sur la lecture et l'exploitation du corpus suivant des réseaux de sens, ce qui, comme nous allons le voir par la suite, prend dans mon travail la forme de trajets thématiques, et cela à travers différents aspects matériels et différentes conditions de production. Cette perspective s'inscrit dans une réflexion autour de la construction des objets d'étude dans l'analyse du discours, telle que l'évoque Pêcheux (1983b : 289 [17]) :

« La langue naturelle n'est pas un outil logique plus ou moins défaillant, mais l'espace privilégié d'inscription de traces langagières discursives, formant une mémoire socio-historique. C'est ce corps des traces que l'analyse de discours se donne comme objet. Par le biais "technique" de la construction de corpus hétérogènes et stratifiés, en reconfiguration permanente, coextensive à leur lecture ».

À l'inverse d'un outil logique, rigide ou stable, l'hétérogénéité apporte à l'analyste la possibilité de repérer dans la mémoire socio-historique les aspects discursifs possiblement intéressants sans que celle-ci soit limitée à une mémoire institutionnelle. Cette reconfiguration permanente, telle que je l'ai mise en place dans mon travail, implique de ne pas se borner de manière rigide à une notion de genre

\footnotetext{
${ }^{\mathrm{f}}$ Au sujet de l'analyse du discours en tant que discipline interprétative, voir Eni Orlandi (1996 [14]).

${ }^{\mathrm{g}}$ L'auteure propose en outre de «suivre un processus de désignation et l'appréhender dans d'autres lieux d'énonciation sans se limiter à la mémoire institutionnelle mais plutôt à la mémoire discursive qui apparaît dans l'archive » (ma traduction).
} 
de discours, mais de se placer dans la perspective dynamique des possibilités ouvertes par les mouvements d'aller-retour entre la problématique et les différents moments (de travail) du corpus (description, analyse et interprétation).

\subsection{Genre de discours : un outil heuristique}

Au départ, j'ai travaillé à l'aide d'une sorte de macro-genre de discours du tourisme ${ }^{\mathrm{h}}$ - celui qui était lié aux guides touristiques - et avec certains sous-genres ou discours qui avaient un rapport avec ces guides. C'est en effet l'idée d'intégrer des discours ayant un air de famille avec ceux des guides qui m'a amené à effectuer l'ouverture dont je parle ici. Je voudrais montrer ici le fonctionnement de ce (macro)genre de discours touristique comme critère de classification et de sélection pour le travail méthodologique de la construction du corpus. J'avais donc à ce moment-là rassemblé le matériel suivant :
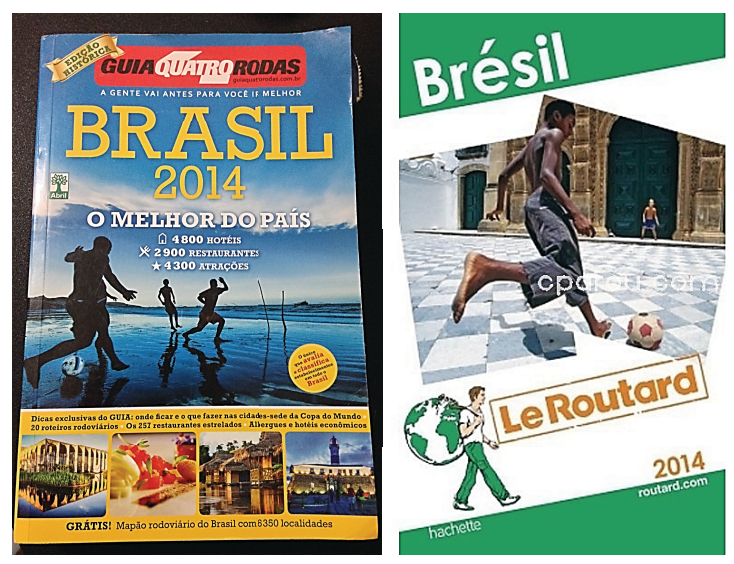

Figure 1. Guides touristique en français et en portugais.

\section{Forum de discussions en français ${ }^{i}$}

Je n'ai pas complété la liste avec des forums de discussion en portugais. Cela a été précisément le moment où j'ai réaménagé mon entrée d'analyse et où j'ai intégré d'autres matérialités qui n'étaient pas prévues au départ. À partir de ce premier regroupement (matériel), j'ai pu en effet vérifier que les énoncés iconico-verbaux dans ce type de matériau se regroupaient déjà autour de ce qui est devenu, par la suite, le thème dans la constitution du corpus, à savoir le sujet de la Coupe du Monde et des voyages réalisés dans ce contexte, tant par les Brésiliens que par les étrangers. Au lieu d'intégrer des forums de discussions en portugais, dont la circulation ne se fait pas comme en France (par exemple dans le cas d'un forum lié à un guide, comme c'est le cas pour le Routard), j'ai intégré les lieux numériques liés aux ministère du tourisme brésilien : en effet, $\mathrm{j}$ 'ai pu observer qu'au niveau du thème (Coupe du Monde) aussi bien qu'au niveau de la production/circulation d'une masse considérable de discours, c'était plutôt ces lieux que je devrais privilégier. Cela s'est matérialisé effectivement dans le changement de l'entrée d'analyse et d'un des critères de sélection : les guides en tant que genre de discours ont cédé leur place aux questions thématiques sur la Coupe du Monde.

\footnotetext{
${ }^{\mathrm{h}}$ Je ne rapproche pas la catégorie «genre de discours » (voir partie 2, page 3) de la notion-concept théorique «formation discursive », qui n'est pas traitée dans le présent texte. Je me place dans la perspective théorique de l'analyse du discours, que je dirais «brésilienne »- par rapport à quelques caractéristiques liés à une des analyses du discours «française» qui se font en France aujourd'hui, sur ce sujet voir Paveau 2012 [18]. Dans la perspective matérialiste de l'analyse du discours (brésilienne), on suit les considérations faites dans les études du telles qu'elles sont synthétisés dans Courtine, 1981 [19], parmi d'autres.
}

i http://www.routard.com/comm_forum_de_voyage.asp 


\section{Reformulation de ma question: du genre de discours au trajet thématique}

Ayant utilisé comme point de départ le genre de discours en tant que critère flexible pour filtrer la circulation des discours, je suis ensuite passée à l'utilisation d'une autre entrée pour la constitution du corpus : le trajet thématique. Le thème des voyages au Brésil lors de la Coupe du Monde de football 2014, est alors devenu un événement, dans le sens défini par Moirand (2007 [20]) ; le concept de thème est celui à partir duquel j'ai continué mon travail de constitution de corpus. Je parlerai donc du trajet thématique et de la notion d'événement, pour expliciter, par la suite, la (re)configuration du corpus et par conséquent de certains de mes critères d'analyse.

\subsection{Trajet thématique et événement discursif}

Le genre est donc conceptualisé à partir de la notion d'événement discursif, en utilisant le trajet thématique comme dispositif de lecture de l'archive. Ce sont les trois notions-clé que j'ai pu mettre en évidence au moment où j'ai finissais l'étape de la classification/sélection des documents et où je suis passée à la description/analyse/interprétation. Quoiqu'étant encore dans une étape intermédiaire de la constitution (analytique) du corpus, il m'est possible d'identifier la façon dont a été articulée la notion d'événement discursif et quel rôle a eu le trajet thématique dans cette étape.

Cette réflexion s'appuie en particulier sur deux articles déjà mentionnés de Zoppi-Fontana (2003 [1], 2005 [21]) qui reprend pour sa part les notions mises en place par Guilhaumou \& Maldidier (1989 [22]) et Guilhaumou, Maldidier \& Robin (1994 [7]) :

«La notion de thème ne renvoie ici ni à l'analyse thématique telle qu'elle est pratiquée par les littéraires, ni aux emplois qui en sont faits dans la linguistique. Elle suppose la distinction entre "l'horizon d'attente" l'ensemble des possibles attestés dans une situation historique" - et l'événement discursif qui réalise un de ces possibles, inscrit le thème en position référentielle ». (Guillaumou \& Maldidier. Effets de l'archive, dans Guilhaumou, Maldidier \& Robin (1994 : 93 [7]).

Selon ces auteurs, l'événement discursif « est à saisir dans la consistance d'énoncés qui font réseau à un moment donné » (Guillaumou \& Maldidier, 1994 : 93 [7]). Ainsi, si au départ je parlais d'un genre de discours - comme celui du tourisme - «le voyage vers le Brésil » peut être identifié comme le thème à partir duquel j'ai entamé la constitution du corpus. Ce thème s'est spécifié dans les premiers moments d'analyse, comme on le verra par la suite. À partir de l'inscription du thème dans l'actualisation d'une de ses possibilités, il est possible de mettre en place l'analyse.

«Si l'analyse d'un trajet thématique repose sur la connaissance des traditions rhétoriques, des formes d'écriture, des usages langagiers, elle met avant tout l'accent sur le nouveau dans la répétition. Une telle analyse ne s'arrête pas aux limites d'une écriture, d'un genre, d'une série : elle reconstruit les cheminements de ce qui fait événement dans le langagier ». (pp.93-94).

Il est intéressant de voir l'interrelation de la notion de trajet thématique et celle de genre de discours, c'est ainsi que s'est dessiné le parcours que j'ai mené dans mon travail. Dans ce sens, le trajet thématique est celui qui suit le thème des voyages au Brésil pendant la Coupe du Monde, tout cela étant lié aux productions discursives qui m'intéressent, à savoir les propos genrés, racisés, sexistes, stéréotypés et élaborés à partir d'une conception de la mémoire de la colonisation. Autrement dit, le trajet thématique dans mon travail - guidé en outre par une recherche sur la construction, la formulation et la circulation d'une doxa - se présente comme l'ensemble des configurations linguistico-discursives autour des questions des stéréotypes genrés, racisés, post-coloniaux, produits dans le contexte des discours sur les voyages au Brésil pendant la Coupe du Monde 2014. Ce trajet thématique lie voyages au Brésil et Coupe du Monde de football 2014, comme nous pouvons le voir sur l'image 1 ; cela était déjà prévu dans mon projet initial, mais en suivant ce trajet thématique, j'ai intégré une plus large diversité matérielle dans mon corpus, comme par exemple le site du ministère du Tourisme au Brésil. 

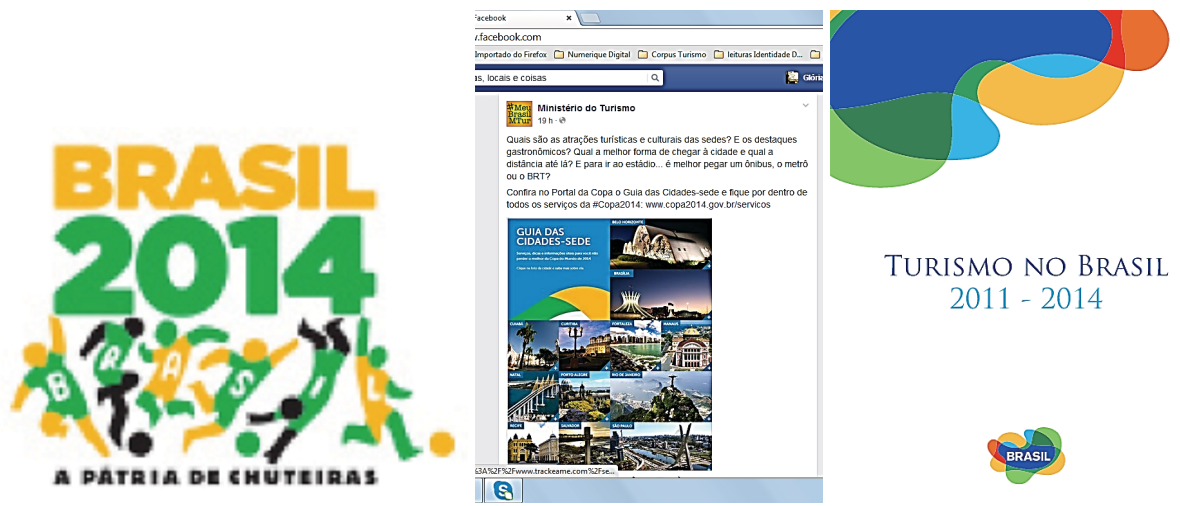

TURISMO NO BRASIL $2011-2014$

Figure 2. (à gauche) Logo du site du ministère du tourisme en 2014.

Figure 3. Diffusion d'une application-guide sur le compte Facebook du Ministère du Tourisme, 2014.

Figure 4. (à droite) Document en PDF diffusé sur le site du Ministère du Tourisme.

Dans la Figure 2 par exemple, nous voyons le compte officiel du Ministère du Tourisme sur le réseau Facebook, qui diffuse un «guide» sur les villes où il y aurait des matchs de la Coupe du monde. Ce guide est une sorte d'application conçue pour être téléchargée sur différents dispositifs numériques, tels que les Smartphones et les tablettes, et s'adressant aux touristes brésiliens ou étrangers. Je ne considère pas qu'il s'agisse tout simplement d'un guide de tourisme sur un autre support : il ne s'agit pas du même genre de discours mais plutôt d'un technogenre (Paveau, 2013 [2]) que l'on peut intégrer dans l'analyse à partir du repérage thématique. L'existence d'un dossier (Tourisme au Brésil - 2011/2014), comme celui illustré dans la figure 3, par exemple, montre bien le parcours de ce trajet thématique «voyages au Brésil lors de la Coupe du Monde », qui ne s'est pas limité aux deux genres de discours prévus au départ (guide de tourisme et forum de discussions).
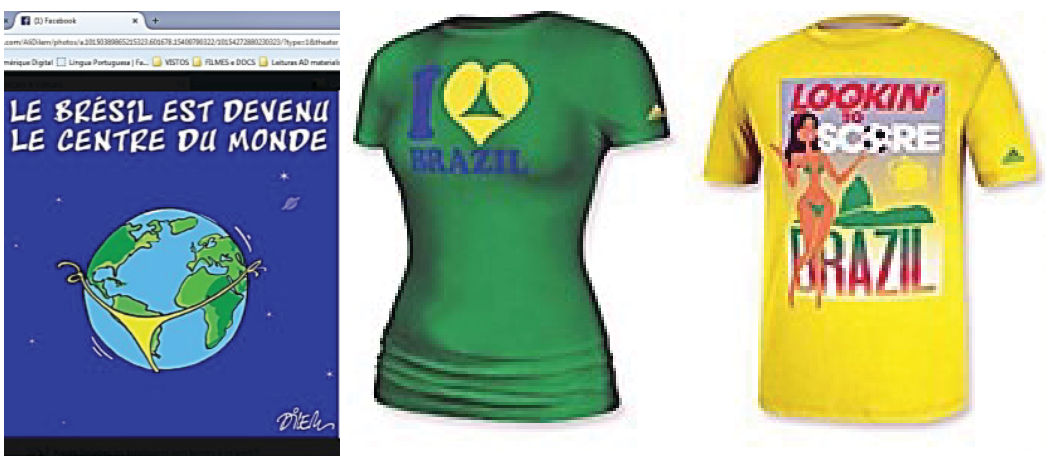

Figure 5. (à gauche) Dessin diffusé pendant Coupe du monde, 2014.

Figure 6. (à droite) T-shirts Adidas mises en vente lors de la Coupe du Monde.

Le travail discursif sur le thème identifié est donc réalisé dans d'autres instances qui dépassent dans le cas du Brésil - ceux liés aux guides de tourisme. À partir de ces exemples, il est possible de saisir l'élargissement de l'hétérogénéité symbolique et institutionnelle permise par le changement d'entrée(s) et de critère(s) dans la constitution du corpus. Cependant, étant donné que ma problématique sert à délimiter mon approche, j'ai dû redéfinir les bornes de ce trajet thématique en le rapprochant de mon objectif de travail sur les propos sexistes, racistes, etc. C'est ainsi que l'un des trajets thématiques, sur lequel j'ai travaillé pendant ma première année, s'est limité aux questions du « tourisme sexuel». 

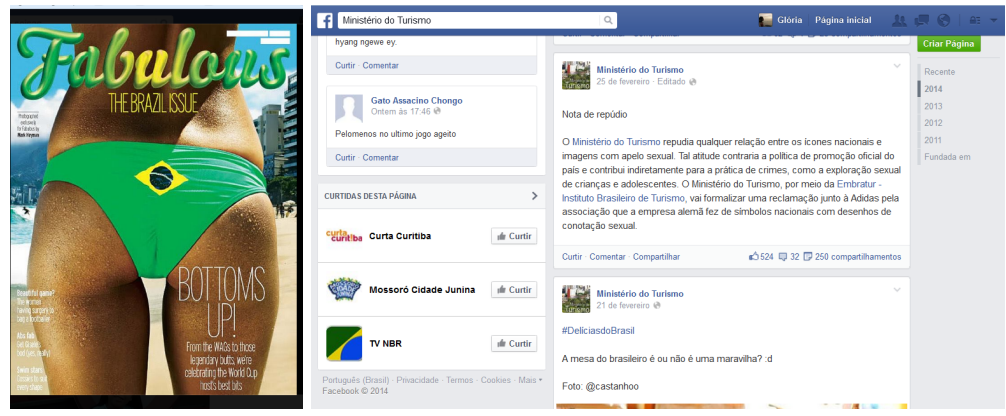

Figure 7 (à gauche). Numéro spécial sur la coupe du monde et sur le « sujet Brésil » Coupe du Monde. Figure 8 (à droite). Notes du Ministère du Tourisme, sur les associations entre l'image du Brésil et des « connotations sexuelles ».

Parler d'un trajet thématique sur la question du tourisme au Brésil lors de la Coupe du Monde permet de définir, à la manière de Guilhaumou et Maldidier le font (1994 : 94 [7]), «l'ensemble des configurations textuelles qui, d'un événement à l'autre associent » plusieurs questions, qui sont, dans mon travail, ceux liés aux thèmes du Brésil, de la Coupe du Monde, du Tourisme sexuel. Tous ces exemples que je montre ne sont pas effectivement dans mon corpus (de travail), mais je les utilise pour illustrer la diversité des genres discursifs qu'on peut rencontrer lorsque l'on fait le choix de suivre un trajet thématique. Ces exemples ont été rassemblés Dans le cas de ces exemples, je les ai rassemblés au tour des (sous-) thèmes, tels que «prostitution », «travesti », « touristes », « tourisme sexuel », «putes », ou encore la présence d'image ou la description des corps considérés comme féminins avec une allusion à la sexualité (c'est le cas pour des publicités, pour certains dessins et pour des publications du ministère du tourisme contre cette image) liés à la question de la coupe du monde. Selon, Zoppi-Fontana (ma traduction, 1999: 3 [6]), le trajet thématique, en tant que dispositif de lecture, permet de mettre en «état de dispersion» des énoncés produits dans des lieux, temps et genres distincts et par des locuteurs différents. Selon cette auteure, cela

«fonctionne comme un fil conducteur, ce qui permet de regrouper des matériels textuels divers dans la construction du corpus, des matériels qui sont à leur tour sélectionnés parce qu'ils font émerger, à partir du fonctionnement des formes linguistiques, dans leur matérialité spécifique, de nouvelles déterminations pour le thème étudié ».

Le trajet thématique contribue ainsi à la construction du corpus à partir de déterminations produites par la matérialité spécifique de la langue dans la discursivité, ce qui permet spécifiquement de souligner les effets de l'événement discursif à l'intérieur de l'archive.

Il me faut préciser que le sens de la notion d'événement discursif telle que l'emploie ZoppiFontana est dérivée du concept développé par Pêcheux (1983a [15]), en particulier dans Le discours : structure ou événement. Selon cet auteur, ce qui fait événement est pris dans ce qui peut déstructurerrestructurer les réseaux de mémoire :

«Il ne s'agit pas de prétendre ici que tout discours serait comme un aérolithe miraculeux, indépendant des réseaux de mémoire et des trajets sociaux dans lesquels il fait irruption, mais de souligner que, par sa seule existence, tout discours marque la possibilité d'une déstructuration-restructuration de ces réseaux et trajets : tout discours est l'indice potentiel d'un bougé dans les filiations socio-historiques d'identification, dans la mesure où il constitue à la fois un effet de ces filiations et un travail [...] de déplacement dans leur espace $[\ldots] »$.

Dans cette conception utilisée par Zoppi-Fontana et par Pêcheux, l'on ne peut identifier un événement discursif que lors de l'analyse-interprétation, et ainsi, même s'il est possible qu'un événement apparaisse dans mon travail, je ne pourrais pas l'identifier a priori. Pour le moment, ce que j'ai réalisé dans mon travail va plutôt dans le sens de ce qui a été exploité dans les divers travaux de Moirand (2007 [20]), qui considère l'événement en tant que production discursive et médiatique concernant un fait du monde réel. Ainsi, un événement peut devenir, à partir des médias un instant discursif ou bien un moment discursif :

« on tente de saisir la diversité des productions discursives qui surgissent, parfois brutalement, dans les médias, à propos d'un fait du monde réel qui devient par et dans les médias un «événement » : par exemple, la coupe du monde de football de 1998, les tempêtes de décembre de 1999, [...] Mais un fait ou un événement 
ne constitue un moment discursif que s'il donne lieu à une abondante production médiatique et qu'il en reste également quelques traces à plus ou moins long terme dans les discours produits ultérieurement à propos d'autres événements » (Moirand, $2007: 3-5$ [20]).

À ce sujet, Dela-Silva (2008 [23]) propose une typologie qui va, je pense, dans le même sens que ce que propose Moirand. Dela-Silva (ma traduction, $2008: 27$ [23]) parle d'événement historique qui, sous un regard discursif, peut être compris « en tant qu'événement de l'ordre de la réalité, des pratiques humaines, peut être discursivisé de différentes façons et produire divers effets de sens ». Dans cette même perspective discursive, Dela-Silva déplace la notion d'événement journalistique de la simple notion de fait (telle qu'il est conçu et défini par les sciences de la communication) à celle d'un «fait que le journaliste considère d'intérêt journalistique », ce qui n'est pas la même chose, puisque le journaliste produit le sens des événements qu'il choisit de mettre en valeur. Je pourrais donc dire que dans la notion de trajet thématique, qui est une notion qui se place finalement au cœur de la réflexion présentée ici, le travail élaboré par l'identification des «moments discursifs » pendant la Coupe du Monde, est proche de ce qui est identifié par Dela-Silva comme événement journalistique.

\subsection{La configuration thématique du corpus}

J'ai ainsi abouti à un trajet thématique autour de l'événement «tourisme sexuel pendant la coupe du monde », et j'ai rassemblé une masse des discours et techno-discours circulant à ce moment discursif. Il est possible d'identifier ce moment puisque ça parle ${ }^{j}$ pendant une période considérable : avant la Coupe du Monde (notamment à travers le lien effectué entre Coupe du Monde et prostitution); avant et pendant la Coupe du Monde (à travers une campagne du Ministère du tourisme, invitant les agents de voyages et la population à dénoncer ce qu'ils appellent «l'exploitation des enfants et des adolescents » par exemple); pendant et après la Coupe du Monde - avec notamment un lieu d'énonciation intégré en cours d'analyse : celui des travailleur.se.s du sexe. À ce sujet, les travailleur.se.s du sexe ont fait une manifestation contre l'amalgame entre prostitution et exploitation sexuelle, et il a ainsi été possible d'observer un vrai «événement» discursif (dans le sens donné par Pêcheux de déplacement de sens stabilisés) : on voyait circuler des discours autour de l'énoncé «le tourisme sexuel est légal» pendant une manifestation de prostitués en raison de la fermeture du bâtiment où ils/elles travaillaient une semaine avant le début de la coupe du monde. L'observation de ce découpage illustre bien que cela ne peut être analysé que lorsqu'on utilise la notion d'archive, qui donne un support à la comparaison et à la constitution d'un sous-corpus à la fois hybride et hétérogène.

Considérer ces différentes matérialités selon un continuum va dans le sens d'une conception dynamique du corpus, telle que nous l'avons définie antérieurement. Cette permanente construction du corpus rend possible, selon Zoppi-Fontana (2003: 3 [1]), de « décrire les régimes d'énonciabilité dans sa dispersion, tant dans ses régularités de fonctionnement que dans les ruptures provoquées par l'événement». L'analyste effectue ainsi des mouvements en spirale qui sont des processus de description aux processus d'interprétation : cela m'a permis (pour ma part) d'incorporer de nouveaux éléments au corpus initialement constitué.

S'il est clair que tous les énoncés ne peuvent pas entrer directement dans l'analyse, il est néanmoins important de les intégrer à mon corpus de référence/archive, ce qui peut m'aider à justifier la composition (matérielle) finale de mon corpus, constitué de la manière suivante :(a.) guides en français et en portugais ; (b.) forums de discussions en français; (c.) comptes Twitter et Facebook du ministère du tourisme brésilien; (d.) site officiel du ministère du tourisme; (e.) blogs de voyages en portugais et en français. Une telle composition n'est pas un a priori analytique dans la théorie du

\footnotetext{
j "Ça parle" est une référence lacanienne très souvent reprise en analyse du discours pour indiquer le travail de l'idéologie dans le discours. Cette formule (ça parle) apparait par exemple dans le travail de Courtine, déjà cité dans ce texte, lorsque cet auteur définit la notion de formation discursive et cite, dans son travail, un extrait de Pêcheux: « le propre de toute FD est de dissimuler, dans la transparence du sens qui s'y forme, (...) le fait que «ça parle » toujours « avant, ailleurs, ou indépendamment » (Pêcheux, 1975, apud Courtine, $1981: 34$ [19]).
} 
discours, bien au contraire : comme nous l'avons vu, il existe des liens entre ces différents genres qui sont en soi constitutivement hétérogènes. Je vais donc toujours conserver la notion de genre de discours va toujours être conservée dans mon travail, et il est possible que dans un deuxième moment je la convoque en vue d'observer en détail si la circulation des stéréotypes sexistes, racistes, postcolonialistes, fait partie de la routine d'un genre plutôt que d'un autre.

\section{Bibliographie}

1. M. Zoppi-Fontana, Identidades (in)formais. Contradição, processos de designação e subjetivação na diferença. ORGANON, 17, n.35, p.45-282, (jan/dez 2003)

2. M-A. Paveau, Technologies discursives [Carnet de recherche], http://technodiscours. hypotheses.org/? $p=311$

3. M. Pêcheux, "Rôle de la mémoire". In : P. Achard et al. (dir), Histoire et Linguistique. (Paris, Éd. de la Maison des Sciences de l'Homme, 1984).

4. M. Zoppi-Fontana, "Cidade e discurso: paradoxos do real, do imaginário, do virtual". RUA Revista do Núcleo de Desenvolvimento da Criatividade, 4, p. 39-54, (1998)

5. M. Pêcheux, "Lire l'archive aujourd'hui", Archives et documents de la SHESL, $\mathrm{n}^{\circ}$ 2, (ENS SaintCloud, Paris, 1982).

6. M. Zoppi-Fontana, "Ordem jurídica, ordem política e a (des)ordem nas ruas". Discurso y Sociedad, 1[1], p. 105-122, (1999)

7. J. Guillaumou, D. Maldidier, R. Robin. Discours et archive. Expérimentations en analyse du discours. (Paris, Mardaga, 1994)

8. M. Zoppi-Fontana, "Acontecimento, arquivo, memória: às margens da lei", Leitura . (LCV/CHLA/UFAL, Maceió, 2002)

9. S. Moirand, L'impossible clôture des corpus médiatiques. La mise au jour des observables entre catégorisation et contextualisation, Revue Tranel, 40, p. 71-92, Neuchâtel (2004.)

10. E. Orlandi, Segmentar ou Recortar ?, Lingüística: Questões e Controvérsias, 10. (Uberaba, 1984, p.9-26).

11. M. Zoppi-Fontana, Disciplina: Autoria, Efeito-leitor e gêneros de discurso, REDEFOR (São Paulo, 2012, version non publiée)

12.S. Moirand, Le même et l'autre dans les guides de voyage au XXIe siècle. La communication touristique. Approches discursives de l'identité et de l'altérité. (Paris : L'Harmattan, p. 151 - 172, 2004b)

13. M. Zoppi-Fontana, Lugares de enunciação e discurso. Leitura-Análise do Discurso. Revista do Programa de Pós-graduação em Letras e Lingüística, 23, p. 15-24, (jan/jun [1999], 2002).

14. E. Orlandi, Interpretação: autoria, leitura, efeitos do trabalho simbólico. (Petrópolis: Vozes, 1996)

15. M.Pêcheux, "Le discours: structure ou événément ? " In : D. Maldidier, L'inquiétude du discours (Cendres, Paris, [1983a] 1990.)

16. J. Guillaumou, "Le corpus en analyse de discours : perspective historique", Corpus [En ligne], 1. consulté le 05 mai 2013. URL : http://corpus.revues.org/8 (2002)

17. M. Pêcheux, "Lecture et mémoire: projet de recherche. " In: D. Maldidider, L'inquiétude du discours (Cendres, Paris, [1983b] 1990)

18. M-A. Paveau, "Que veut dire travailler en analyse du discours en France en 2011 ? Épistémologies, objets, méthodes", actes du colloque III Encontro Internacional de Estudos da Linguagem, septembre 2011; Pouso Alegre, en ligne, 2012)

19. J-J. Courtine, "Quelques problèmes théoriques et méthodologiques en analyse du discours, à propos du discours communiste adressé aux chrétiens". Langages, 61, 1981.

20. S. Moirand, Les discours de la presse quotidienne. Analyser, comprendre, (PUF, Paris, 2007)

21. M. Zoppi-Fontana, "Arquivo jurídico e exterioridade. A construção do corpus discursivo e sua descrição/interpretação", In: E. Guimarães e M. R. Brum de Paula. Memória e sentido. Santa Maria, UFSM/PONTES, p.93-116, (2005) 
22. J. Guillaumou \& D. Maldidier. De l'énonciation à l'événement discursif en analyse du discours. Histoire, épistémologie, langages. 8 (1989).

23. S. Dela-Silva, O acontecimento discursivo da televisão no Brasil: a imprensa na constituição da TV como grande mídia. Tese Unicamp, (2008). 